\title{
Let's urgently engage ourselves in "greening" endoscopy to address ecological issues!
}

\section{다)(우 $\odot$}

\author{
Authors \\ Mathieu Pioche ${ }^{1}$, Thomas Lambin ${ }^{2}$, Jérôme Rivory ${ }^{1}$ \\ Institutions \\ 1 Endoscopy Unit - Digestive Disease Department, \\ Edouard Herriot Hospital, Lyon, France \\ 2 Adult Gastroenterology, Lille University Hospital, Lille, \\ France
}

\section{Bibliography}

Endosc Int Open 2021; 09: E1752-E1753

DOI 10.1055/a-1546-8975

ISSN 2364-3722

(C) 2021. The Author(s).

This is an open access article published by Thieme under the terms of the Creative Commons Attribution-NonDerivative-NonCommercial License, permitting copying

\author{
and reproduction so long as the original work is given appropriate credit. Contents \\ may not be used for commercial purposes, or adapted, remixed, transformed or \\ built upon. (https://creativecommons.org/licenses/by-nc-nd/4.0/) \\ Georg Thieme Verlag KG, Rüdigerstraße 14, \\ 70469 Stuttgart, Germany \\ Corresponding author \\ Dr. Mathieu Pioche, Endoscopy Unit - Digestive Disease \\ Department, Pavillon L - Edouard Herriot Hospital, 5 Place \\ d'arsonval, 69437 Lyon Cedex, France \\ Fax: +33472110147 \\ mathieu.pioche@chu-lyon.fr
}

Many disciplines have already integrated an ecological dimension into their research projects by, for example, comparing the environmental impact of several given strategies in order to choose the most ecological one [1-3]. In the field of endoscopy, the introduction of this dimension into our research projects is still in its infancy [4], although our impact on the environment is major. Indeed, we are responsible for many sources of pollution. The amount of waste is much bigger than the simple volume of our devices (stent packaging for example), endoscope disinfection products are polluting, our water consumption is extremely high, rare metals are everywhere in our equipment, and there is talk of introducing disposable endoscopes for which the ecological impact has not yet been assessed [5]. Furthermore, we use animals for our training without any evidence demonstrating the benefits of living models compared to isolated organs.

In contrast, urologists [6] and anesthetists [7] have introduced ecological dimensions into their evaluations from the outset and surprisingly, they have shown that disposable endoscopes (which have less plastic than our own scopes) have less environmental impact than reusable versions. This interesting finding underscores the complexity of ecological evaluation. When we consider the entire production chain from the factory to recycling (including packaging, transport, and decontamination), reusable solutions may paradoxically be more polluting than disposable ones.

Thus, it is already possible to fight waste while keeping in mind that global evaluations are necessary because the problem is extremely complex. For example, is it better to use a dual cold/hot snare for all patients to favor a single tool or to use a pure cold snare in most cases and an additional hot snare only when necessary? Indeed, simplicity could push us to favor a single tool, but what if the pollution generated by the electrical part is greater than that from a pure cold device? All of this requires well-conducted evaluations that attempt to measure the problem in a global way.

So far, clinical trials and guidelines from endoscopy societies have focused on the clinical benefit of a strategy that balances possible adverse events and costs without integrating an ecological dimension into the choice of therapeutic option. Should we reconsider this widespread dogma? For example, the comparison of multiple plastic stents every 3 months versus single self-expandable metal stents for 9 months for the treatment of biliary strictures could integrate environmental impact issues and that could change the strategy chosen. If the clinical benefit is the same between strategies, should we consider choosing the most sustainable one instead of the less expensive one?

Many other aspects can already be considered without a research protocol. Waste sorting and recycling in hospitals remain very complex with soiled equipment, but the lack of sorting runs contrary to the efforts we make every day to be ecoresponsible in our homes. Therefore, it is difficult to rationalize the choice not to apply similar measures in the hospital. For example, in our unit, only cardboard boxes are separated and sent directly for recycling apart from other waste created during patient care, which is not enough. The legal recycling requirements must also be applied to non-soiled medical devices if we are to make progress. 
It just takes common sense on a daily basis to reduce our waste and have less environmental impact. In our endoscopy unit, nurses have proposed simple measures, such as the use of anti-reflux valves for endoscope washing tubes and injection tubes for dissection knives. These small devices, which contain less than $5 \mathrm{~g}$ of plastic, allow us to switch from one plastic tube per patient to one tube per day, considerably reducing the number of water bags and indigo-carmine ampoules we use. To go even further, improvements in endoscopic image storage systems for photos and videos simplify recording but create huge databases of images, the majority of which are useless. Should start recommending selection of key images that contain the necessary information for a patient, without accumulating hours of video that will probably never used but that produce a lot of heat in computer networks? The COVID-19 pandemic has revolutionized our use of digital technologies and made possible beautiful virtual congresses, like the 2021 European Society of Gastrointestinal Endoscopy days. Certainly, human connection is missing, but is it necessary that we once again fly regularly to conferences on the other side of Europe? There are probably intermediate solutions, as our urological colleagues have proposed to keep some face-to-face moments without emitting all the $\mathrm{CO}_{2}$ associated with traditional conference travel.

Manufacturers also must be involved in this change in paradigm. Our message to them must be clear and unanimous in the direction of waste reduction by encouraging them to minimize the amount of waste. We can choose the least polluting device by requiring traceability of the carbon dioxide impact when comparing several competing devices. In view of the evolution of thinking, are we going to introduce a medico-ecological dimension to our studies by creating an additional acceptable price to pay for reducing our waste, similar to what already exists in terms of medico-economic efficiency measures, with the price acceptable to improve survival or quality of life?

We are by no means role models, and even less so, examples, as we have never produced any science on environmental impacts of endoscopy. However, this editorial aims to propose the creation of a community of eco-endoscopists who share simple ideas to bring about a gradual change in the way we do things and will devote themselves to research projects aimed at reducing the impact on the planet. We need to be united in adding this dimension to our assessments. Scientific societies and brands must be integrated into this process to bring more sustainability to our practices.

\section{Competing interests}

The authors declare that they have no conflict of interest.

\section{References}

[1] Tauber J, Chinwuba I, Kleyn D et al. Quantification of the Cost and potential environmental effects of unused pharmaceutical products in cataract surgery. JAMA Ophthalmol 2019; 137: 1156-1163

[2] Milford K, Rickard M, Chua M et al. Medical conferences in the era of environmental conscientiousness and a global health crisis: The carbon footprint of presenter flights to pre-COVID pediatric urology conferences and a consideration of future options. J Pediatr Surg 2020: doi:10.1016/j.jpedsurg.2020.07.013

[3] Thiel CL, Woods NC, Bilec MM. Strategies to reduce greenhouse gas emissions from laparoscopic surgery. Am J Public Health 2018; 108 : S158-S164

[4] Maurice JB, Siau K, Sebastian S et al. Green endoscopy: a call for sustainability in the midst of COVID-19. Lancet Gastroenterol Hepatol 2020; 5: 636-638

[5] Trindade AJ, Copland A, Bhatt A et al. Single-use duodenoscopes and duodenoscopes with disposable end caps. Gastrointest Endosc 2021; 93: 997-1005

[6] Davis NF, McGrath S, Quinlan M et al. Carbon footprint in flexible ureteroscopy: a comparative study on the environmental impact of reusable and single-use ureteroscopes. J Endourol 2018; 32: 214-217

[7] Sherman JD, Raibley LA, Eckelman MJ. Life Cycle assessment and costing methods for device procurement: comparing reusable and single-use disposable laryngoscopes. Anesth Analg 2018; 127: 434443 